\title{
Medición de la cintura de un haz de luz
}

\author{
Franklin Julca ${ }^{1}$, Ilich Contreras ${ }^{1}$ y Whualkuer Lozano Bartra ${ }^{* 1,2}$ \\ ${ }^{1}$ Facultad de Ciencias Físicas, Universidad Nacional Mayor de San Marcos, \\ Ap. Postal 14-0149, Lima 14, Perú \\ ${ }^{2}$ Facultad de Ciencias Naturales y Matemática, Universidad Nacional del Callao, \\ Bellavista, Callao
}

Recibido 21 agosto 2010 - Aceptado 30 setiembre 2010

Hemos medido los perfiles de la distribución de irradiancia y la propagación espacial de un diodo emisor de luz y de un láser HeNe usando diversas técnicas experimentales. El valor experimental de la cintura del láser fue verificado usando la ley de matrices ABCD.

Palabras claves: Perfil gaussiano, fibra óptica, LED, láser HeNe.

\section{Measurement of a light beam waist}

We have measured both the irradiance distribution and the spatial propagation profiles of a light emitting diode and a HeNe laser using several experimental techniques. The experimental value of the waist laser was verified using the ABCD array law at the laser case.

Keywords: Gaussian profile, optical fiber, LED, He-Ne laser.

\section{Introducción}

Los láseres son la primera alternativa como fuentes de energía para muchas aplicaciones en las ciencias básicas, medicina e industria, debido a que entregan energía en forma de luz con una serie de características y de una manera altamente eficaz. Por eso es importante conocer el comportamiento de algunos de sus parámetros, tales como su, potencia, energía, diámetro, perfil espacial, divergencia y su factor de calidad. Es conocido que en muchas aplicaciones, estos parámetros definen el éxito o fracaso de un experimento, de esta forma, su control y optimización son trascendentales. Por ejemplo, la manera como la potencia es distribuida en un haz láser depende del modo o de la combinación de modos que están circulando en la cavidad láser y, como estos modos son distorsionados por la presencia de elementos ópticos usados en la cavidad. El patrón ISO 11146 especifica el diámetro de un haz láser con una distribución gaussiana, como el punto que corresponde al $13.5 \%\left(1 / e^{2}\right)$ de su máxima intensidad.

Para examinar el perfil de un haz, la técnica más usada es el método de prueba de Foucault (MPF), también conocido como knife-edge[1]. Esta técnica hace una medida indirecta, donde la dependencia que se obtiene de la irradiancia del haz con el desplazamiento transver-

\footnotetext{
*whualkuer@yahoo.com.br
}

sal da como resultado el trazo de una función error[2]. Otros métodos usados son el scan slit[3], el del alfiler o pinhole [4] y el de una fibra óptica acoplado a un piezoeléctrico[5].

En este trabajo caracterizamos los perfiles de dos diodos emisores de luz (LEDs) y el perfil de un láser de HeNe, usando las técnicas MPF y el de la fibra óptica, respectivamente. En el caso del láser, verificamos teóricamente la cintura mínima de su perfil espacial de propagación con la ley de las matrices $A B C D[6]$.

\section{Teoría}

La ley de transformación de un haz Gaussiano propagándose a través de diversos medios puede ser descrita por la matriz $A B C D$, como

$$
q_{\alpha}^{\prime}=\frac{A_{\alpha} q_{\alpha}+B_{\alpha}}{C_{\alpha} q_{\alpha}+D_{\alpha}}
$$

donde $A_{\alpha}, B_{\alpha}, C_{\alpha}, D_{\alpha}$ con $\alpha=1,2,3, \ldots, n$, describen los n medios por el cual se propaga el haz. El parámetro $q_{\alpha}$ caracteriza al haz de entrada y el parámetro $q_{\alpha}^{\prime}$ el haz de salida. Los elementos de la matriz $A_{T}, B_{T}, C_{T}$, $D_{T}$ relacionan el plano de salida del último medio y el plano de entrada del primer medio, es el producto de 
las $n$-ésimas matrices que caracterizan los medios de la secuencia, de la forma

$$
\begin{aligned}
\left(\begin{array}{cc}
A_{T} & B_{T} \\
C_{T} & D_{T}
\end{array}\right)= & \left(\begin{array}{cc}
A_{2} & B_{2} \\
C_{2} & D_{1}
\end{array}\right)\left(\begin{array}{ll}
A_{1} & B_{1} \\
C_{1} & D_{1}
\end{array}\right) \cdots \\
& \cdots\left(\begin{array}{ll}
A_{n} & B_{n} \\
C_{n} & D_{n}
\end{array}\right)
\end{aligned}
$$

La ventaja de usar esta matriz $A B C D$ es que nos permite hallar el parámetro del haz gaussiano $q$ a través de una serie de elementos ópticos.

Conociendo que una onda gaussiana puede expresarse como[7]

$$
\exp \left[i\left(\frac{k r}{2 q}\right)\right]=\exp \left[-i\left(\frac{k r^{2}}{2 R}\right)-\left(\frac{r^{2}}{w^{2}}\right)\right]
$$

donde $k$ es el número de onda, $R$ el radio de curvatura del frente de onda, y $w$ la cintura del haz, podemos escribir el inverso del parámetro $q$ como

$$
\frac{1}{q}=\frac{1}{R}-i \frac{\lambda}{\pi w^{2}}
$$

Escribiendo la matriz $A B C D$ que corresponde al producto de las matrices que definen a dos medios, tal como el aire y una lente delgada, obtenemos

$$
\begin{aligned}
\left(\begin{array}{ll}
A & B \\
C & D
\end{array}\right) & =\left(\begin{array}{cc}
1 & d \\
0 & 1
\end{array}\right)\left(\begin{array}{cc}
1 & 0 \\
-1 / f & 1
\end{array}\right) \\
& =\left(\begin{array}{cc}
1-d / f & d \\
-1 / f & 1
\end{array}\right)
\end{aligned}
$$

donde $f$ es la distancia focal y $d$ es la distancia que viaja el haz en el aire después de salir de la lente. Usando la Ec.(1), podemos encontrar que un haz gaussiano propagándose a través de una lente delgada puede ser descrita por

$$
\frac{1}{q_{2}}=\frac{1}{q_{1}}-\frac{1}{f}
$$

donde $q_{1}$ es el parámetro de entrada y $q_{2}$ es el parámetro de salida. Reemplazando la Ec.(4) en la Ec.(6) para la misma lente obtenemos

$$
\frac{1}{R_{2}}=\frac{1}{R_{1}}
$$

considerando que $R_{1} \rightarrow \infty$ y usando la Ec.(4) obtenemos

$$
q_{1}=i \frac{n \pi w_{01}^{2}}{\lambda}
$$

donde $n$ es el índice de refracción de la lente. De la Ec.(1), podemos escribir también

$$
q_{2}=\frac{A q_{1}+B}{C q_{1}+D}=\frac{(1-d / f) i\left(n \pi w_{01}^{2}\right) / \lambda+d}{(-1 / f)-i\left(n \pi w_{01}^{2}\right) / \lambda+1}
$$

separando la parte real y compleja de $q_{2}$, obtenemos

$$
\begin{aligned}
q_{2}= & \frac{d-(1 / f)\left(n \pi w_{01}^{2} / \lambda\right)^{2}+d\left(1 / f^{2}\right)\left(n \pi w_{01}^{2} / \lambda\right)^{2}}{1+\left(1 / f^{2}\right)\left(n \pi w_{01}^{2} / \lambda\right)^{2}} \\
& +\frac{i\left(n \pi w_{01}^{2} / \lambda\right)}{1+\left(1 / f^{2}\right)\left(n \pi w_{01}^{2} / \lambda\right)^{2}} .
\end{aligned}
$$

Tomando la parte real de la Ec.(10) obtenemos

$$
d=\frac{f}{1+\left[f /\left(n \pi w_{01}^{2} / \lambda\right)\right]^{2}},
$$

de la misma tomando la parte imaginaria de la Ec.(10) podemos escribir el parámetro

$$
q_{2}=i \frac{n \pi w_{01}^{2}}{\lambda}=\frac{i\left(n \pi w_{01}^{2} / \lambda\right)}{1+(1 / f)^{2}\left(n \pi w_{01}^{2} / \lambda\right)^{2}} .
$$

De la ecuación (12), obtenemos la cintura mínima del haz

$$
w_{02}^{2}=\frac{w_{01}^{2}}{1+(1 / f)^{2}\left(n \pi w_{01}^{2} / \lambda\right)^{2}} .
$$

\section{Resultados y discusión}

El montaje usado en este experimento es mostrado en la figura 1. En primer lugar, los haces de una fuente de luz que puede ser de un LED o de un láser de HeNe, son alineados paralelamente a la superficie de la mesa óptica usando dos aberturas circulares. Estos haces son colimados y posteriormente expandidos usando un arreglo óptico que consiste de dos lentes con distancias focales de $50 \mathrm{~mm}$ y $150 \mathrm{~mm}$, las cuales no son mostradas en la figura.

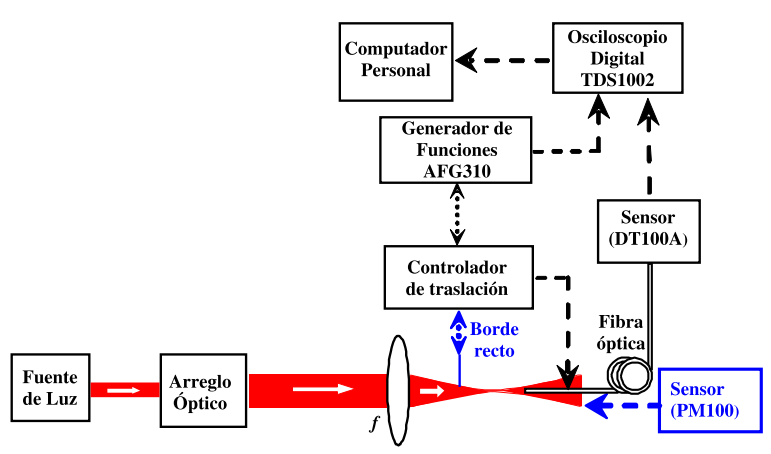

Figura 1: (Color en la versión digital) Diagrama del montaje experimental para ambos casos: para el MPF solo es necesario el borde recto que es operado con el controlador de traslación y el detector PM100 (de azul, en versión digital); en caso del método con la fibra óptica se muestra el esquema completo.

Finalmente, este haz expandido fue focalizado usando una lente de $75 \mathrm{~mm}$. Para medir la cintura del haz 
usando la técnica MPF correspondiente al caso de los LEDs, usamos una lámina fina para bloquear gradualmente el camino del haz hacia el medidor de potencia. Para esto, la lámina fue posicionada transversalmente al haz y montada sobre un trasladador micrométrico XZ.
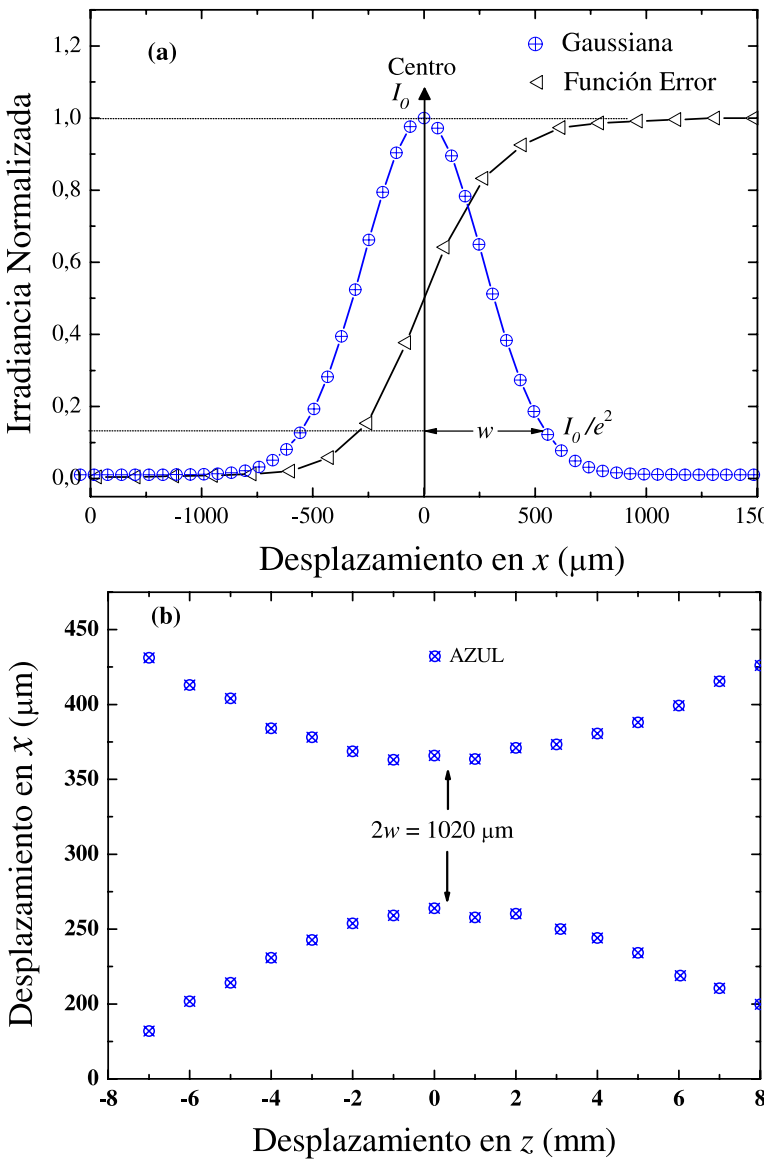

Figura 2: (a) El perfil de la distribución de la irradiancia $I$ es mostrado en dos dimensiones com función del desplazamiento $x$. (b) Típico perfil de propagación espacial de un haz, el gráfico corresponde al perfil de propagación de un LED azul de $471 \mathrm{~nm}$, con $w=510 \mu \mathrm{m}$.

Las medidas transversales de potencia de estos haces, son realizadas usando un medidor de potencia PM100 de la empresa Thorlabs. Para la medición de la cintura del haz del láser, nos basamos en el trabajo de la Ref.[5], con la diferencia que ellos usaron un motor de paso en su experimento y nosotros un piezoeléctrico. Haciendo de esta forma la técnica de medición mucho más simple y de un costo mucho más bajo. El uso de la fibra óptica es debido a que el tamaño del núcleo (8 $\mu \mathrm{m})$ de la fibra es mucho menor que el ancho del haz, y porque se adapta con la estructura de un agujero de alfiler. En la Fig.1, se muestra la fibra óptica acoplada al piezoeléctrico (altavoz o parlante), el cual fue modulado en amplitud y frecuencia por un generador de funciones AFG310 Tektronix. La luz láser en la salida de la fibra fue dirigida a un detector DET100A de la Thorlabs, el cual estuvo conectado a un osciloscopio TDS1002 Tektronix y este a un computador personal para la adquisición de datos.

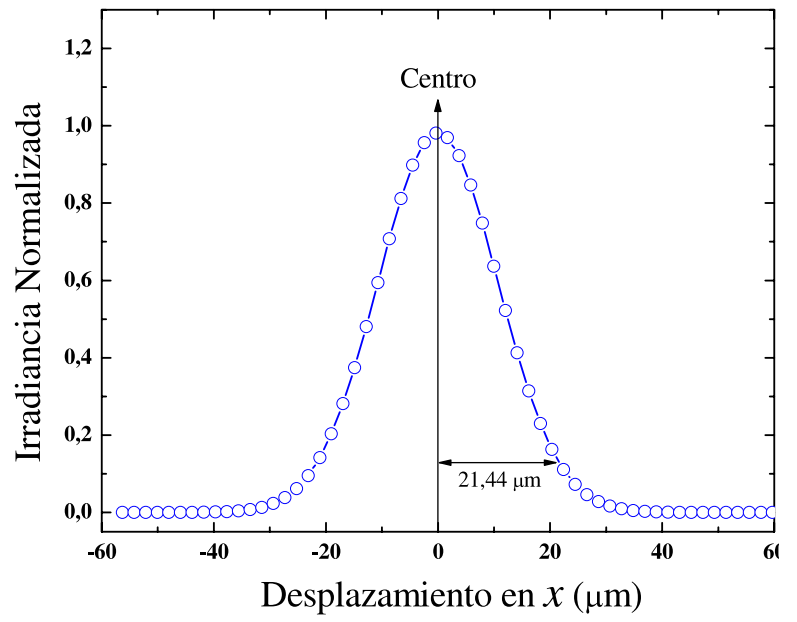

Figura 3: Distribución de la irradiancia usando el método de la fibra óptica. La medida de $21.44 \mu \mathrm{m}$ corresponde a la cintura mínima del láser.

En la experiencia del MPF, realizamos mediciones del haz colimado de un LED rojo $(653.5 \mathrm{~nm})$ y un LED azul $(471 \mathrm{~nm})$. Debido a que los perfiles de distribución de ambos LEDs son prácticamente iguales, mostraremos en la figura 2 los resultados correspondientes al LED azul. En la figura 2a, se puede observar que la dependencia del desplazamiento transversal versus la irradiancia integrada, la cual está indicada por los puntos triangulares, tiene la forma de una función error. La derivada de esta función error, representados por los puntos circulares, muestra el perfil gaussiano del haz de luz. La Figura 2b, muestra el perfil de propagación espacial obtenido al proyectar las cinturas de la gaussiana versus desplazamientos de 1,00 $\mathrm{mm}$ en la misma dirección $z$ del haz. La cintura $w_{0}$ obtenida para el haz del LED rojo fue de $556 \mu \mathrm{m}$ y para el haz del LED azul fue $510 \mu \mathrm{m}$, manifestando que la cintura de un haz depende de la longitud de onda de la radiación que pasa por el lente convergente. También medimos la cintura del haz del láser de HeNe $(632.8 \mathrm{~nm})$ con la técnida MPF, obteniendo una cintura para el haz láser de $w_{0}=24.8 \mu \mathrm{m}$. En este caso, el haz originalmente colimado primero fue expandido y luego focalizado usando lentes con distancias focales de $50 \mathrm{~mm}, 150 \mathrm{~mm}$ y 75 $\mathrm{mm}$, respectivamente. 
Para medir la cintura del haz láser usando una fibra óptica, se procedió primero a medir el diámetro del haz colimado por reflexión sobre una pantalla blanca, obteniendo el valor de $w_{01}=7 \mathrm{~mm}$. Esta medida es relevante para el cálculo teórico de la cintura de un haz láser usando la ley de matrices $A B C D$. Se realizaron diversos desplazamientos a lo largo del eje $z$ para determinar la posición correcta de la cintura mínima $w_{02}$ del haz láser. Tomando el índice de refracción del aire como $n=1$, la longitud de onda central del láser de HeNe como $\lambda=632.8 \mathrm{~nm}$, la distancia focal de la lente como $f=75 \mathrm{~mm}$ y usando la Ec.(13) obtenemos el valor teórico de la cintura del haz $w_{02}=21.38 \mu \mathrm{m}$.

\begin{tabular}{cc}
\hline Cintura del haz de laser & $w(\mu \mathrm{m})$ \\
\hline Teórica & 21.38 \\
MPF & 24.80 \\
Fibra óptica & 21.44 \\
\hline
\end{tabular}

Tabla 1: Valores de las cinturas mínimas obtenidos por los dos métodos experimentales y por el método teórico de las matrices $\mathrm{ABCD}$.

En la figura 3, mostramos el oscilógrama de un perfil gaussiano producido por la modulación del piezo- eléctrico cuando es acoplada la luz láser dentro de la fibra óptica. La cintura mínima para esta distribución gaussiana fue de $21,44 \mu \mathrm{m}$. La Tabla 1 , muestra el valor teórico y los valores experimentales encontrados usando las dos técnicas mencionadas.

\section{Conclusiones}

Hemos obtenido el valor del perfil de distribución de la irradiancia y del perfil de propagación espacial de dos fuentes de luz tales como un LED y de un láser de HeNe, usando el método MPF. Obtuvimos también el valor de la cintura mínima de un haz láser empleando una fibra óptica. El valor de la cintura mínima del láser fue comparado con el valor encontrado usando la ley de matrices $\mathrm{ABCD}$ y, los resultados fueron muy cercanos, demostrando la eficiencia del método usado.

\section{Agradecimientos}

Este trabajo fue parcialmente financiado por el Consejo Superior de Investigación del Vicerrectorado de Investigación y por el Vicerrectorado Académico a través del Programa de Iniciación Científica.

\section{Referencias}

[1] W. Plass, R. Maestle, K. Wittig, A. Voss y A. Giesen, High-resolution knife-edge laser beam profiling, Opt. Commun. 134, 21 (1997).

[2] Maria Isabel Ribeiro, Gaussian Probability Density Functions: Properties and Error Characterization, Institute for Systems and Robotics (2004).

[3] Jose Soto, Arbitrary intensity profiles measurement of laser beams by a scanning and rotating slit, Appl. Opt. 32, 7272 (1993).
[4] C.H. Kuo, Transverse Profile Measurement System at SRRC, EPAC'94, London (1994).

[5] Fang-Weng Sheu y Ching-Huang Chang, Measurement of the intensity of $s$ Gaussian laser beam near its focus using an optical fiber, Am. J. Phys. 75, 956 (2007).

[6] Amnon Yariv, Quantum Electronics, pp.116-124, Third Edition, John Wiley \& Sons, California (1988).

[7] Javier Alda y Josep Arasa, Encyclopedia of Optical Engineering, pp.999-1011, Taylor \& Francis Group, Londres (2003). 